\title{
Wall-adapting subgrid-scale models to apply to large eddy simulation of internal combustion engines
}

\author{
Andrea Montorfano*, Federico Piscaglia and Angelo Onorati \\ Dipartimento di Energia, Politecnico di Milano, I-20156 Milano, Italy
}

(Received 20 October 2012; revised version received 15 January 2013; accepted 2 March 2013)

\begin{abstract}
In this work a methodology to perform large eddy simulation of in-cylinder flows by the open-source CFD code OpenFOAM ${ }^{\circledR}$ is presented. First, authors implemented the dynamic Smagorinsky and the wall-adapting local eddy-viscosity (WALE) subgrid-scale model in the code; model validation has been performed by comparison against experiments carried out on a standard test case of flow with separation and recirculation. The WALE model has been further applied on a simplified engine-like geometry. Also in this case results look satisfying, although discrepancies still exist between simulations and experiments. An explanation for such a behaviour is suggested in the paper, together with a possible solution.
\end{abstract}

Keywords: large eddy simulation; OpenFOAM; WALE; dynamic Smagorinsky; backward-facing step; LES of engine-like geometry; LES of ICE by OpenFOAM

2010 AMS Subject Classifications: 76F10; 76F65

\section{Introduction}

In large eddy simulation (LES), Navier-Stokes equations are filtered in order to separate larger anisotropic scales that are responsible for the greatest part of mass and momentum transfer, from the smallest scales, which are supposed to be isotropic by virtue of the first Kolmogorov hypothesis $[9,15]$. Larger or 'grid' scales are directly solved, while smaller or 'subgrid' scales (SGS) are represented by an SGS tensor $\tau_{i j}$, which most often is calculated by the eddy-viscosity assumption [16]:

$$
\tau_{i j}^{\mathrm{sgs}}-\frac{1}{3} \tau_{k k}^{\mathrm{sgs}}=-2 \nu_{\mathrm{sgs}} \bar{S}_{i j}
$$

where $\bar{S}_{i j}$ is the filtered rate-of-strain tensor and $v_{\mathrm{sgs}}$ the subgrid viscosity as calculated by a specific model.

One of the earliest SGS models is due to Smagorinsky [18]; it adopted a linear relation between the SGS viscosity and the norm of the filtered rate-of-strain tensor $\sqrt{\bar{S}_{i j} \bar{S}_{i j}}$, where the proportionality coefficient assumed a constant value. The Smagorinsky model behaves very well in homogeneous isotropic turbulence and free-shear flows, but it was found to have an incorrect behaviour in the proximity of solid walls, due to a non-vanishing $v_{\text {sgs }}$ into the laminar sublayer. The use of a blending function [22] may limit this drawback, but its formulation is restricted 
to equilibrium boundary layers. On the other hand, the need for an SGS model that correctly complies with wall-bounded flows is absolutely unavoidable, since gas flow occurring in IC engines is always confined between solid boundaries, either intake/exhaust ducts, valve ports or cylinder walls. A possible solution was found by Germano et al. [6], who employed a dynamic procedure to determine the value of the proportionality coefficient between $v_{\mathrm{sgs}}$ and $\sqrt{\bar{S}_{i j} \bar{S}_{i j}}$, where $s_{i j}$ is the deformation tensor of the resolved field. The model parameter is not constant anymore, rather a function of time and space. Other authors followed a different philosophy, adopting a universal constant but a different formulation of the subgrid viscosity, so that it naturally vanishes at walls. This is the foundation of wall-adapting local eddy-viscosity model (WALE) [12]. However, a correct switch to laminar behaviour into the viscous sublayer is not enough for a complete representation of wall-bounded flows, since the complex cycle of near-wall turbulence dynamics [17] should be resolved as well. This requires a very fine mesh in terms of wall units, namely, $y_{0}^{+}<2, \Delta x^{+}<10$ and $\Delta z^{+}<5$ [16]. Fortunately, good results could be obtained also with less demanding grids: the outer layer can be correctly resolved even with $y_{0}^{+}<2, \Delta x^{+} \approx 100$ and $\Delta z^{+} \approx 20$ [2]. Nonetheless, these requirements can lead to a high computational cost, which makes them inappropriate for industrial applications.

This work aims at investigating the suitability of LES for complex wall-bounded flows, like those occurring in IC engines, adopting wall-adapting SGS models, but in conditions where 'ruleof-the-book' resolution requirements cannot be satisfied. All models used have been implemented by the authors in LibICE $^{\circledR}$, an open-source $\mathrm{C}++$ library based on the OpenFOAM ${ }^{\circledR}$ technology. The models and the set-up described in this paper are intended for cold flow steady simulations of ICE by the open-source CFD code OpenFOAM ${ }^{\circledR}[13,14]$. The work represents a step towards the LES simulation of a real engine cycle: at the moment, the implementation of a novel parallel algorithm for moving mesh with topological changes for ICE simulation and the implementation of the models used here in their compressible formulation is currently over and under testing.

The paper is organized as follows: in Section 3, validation of the SGS models implemented (dynamic Smagorinsky and WALE) is performed on a standard test case, the backward-facing step (BFS) by Eaton et al. [4], which was chosen because its separating and recirculating flows have many similarities with the gas flow through an engine inlet valve. For this case, hot wire anemometry (HWA) measurements of velocity field at several locations throughout the domain were available for comparison [4]. In Section 4, the WALE model has been applied to a simplified engine configuration for which measurements by laser-Doppler anemometry (LDA) were available [21]. The base theory of the SGS models is available from the original papers by Germano et al. [6] and Nicoud and Ducros [12].

\section{Numerical set-up}

Second-order central differencing schemes in space for advection and diffusion were blended with linear-upwind schemes to stabilize solutions while maintaining second-order behaviour [8]. The schemes applied result to be fully conservative and since the coefficients are always positive they are unconditionally bounded. Also, they satisfy the transportiveness requirement for large values of local (cell) Peclet numbers. Unfortunately, no higher order schemes can be applied in the FV context when unstructured grids are used [7,9]. The numerical set-up described in this section will be kept for all the simulations carried out in this work. Time-marching was implicit, with the time derivative being discretized by a second-order backward approximation. The flux terms were explicitly advanced, the provisional velocity field was then corrected via the pressure gradient by a projection onto a divergence-free velocity field, pressure was computed as a solution to the 
pressure-Poisson problem. In order to improve the global convergence of the simulation, the operator splitting technique for pressure-velocity coupling was implemented as a transient-SIMPLE algorithm [23], where convergence of pressure-velocity solution is enforced by iterating the coupling procedure for each time step; the resulting fluid-dynamic Courant-Friedrichs-Lewy (CFL) number, defined as CFL $=|\boldsymbol{u}| \Delta t / \Delta x$ [5], has been set to 10, with significant advantages in terms of the required wall time.

The code and the developed libraries were fully parallelized. The fluid-dynamic information recorded by a Reynolds-Averaged Navier-Stokes simulation on a precursor domain has been used to reconstruct the turbulent fluctuations at the boundary inlet by a synthetic turbulence inlet b.c. [3]. Other techniques could have been used as well $[11,20]$.

\section{Backward-facing step}

\subsection{Case set-up}

The computational domain of the BFS case is represented in Figure 1. The inlet length was chosen to be $L_{1}=10 H$ (where $H$ is the step height) in order to have a completely developed boundary layer at the step. A developed profile is needed at the outflow as well, and the outlet channel length has been chosen accordingly, leading to $L_{2} / W_{2}=12$.

The domain has been discretized with a fully structured hexahedral grid with two refinement levels, leading of a total elements number of approximately 1.1 million for the coarse mesh, and approximately 2.2 million for the fine mesh. Mesh resolution near the walls turned out to be $y_{0}^{+}=0.8$ for the coarse mesh and 0.1 for the fine mesh, $\Delta x^{+} \in(150 \div 350)$ and $\Delta z^{+} \approx 60$. Even though the first off-wall mesh points were placed well into the laminar sublayer, streamwise and spanwise cell sizes are far too large for a complete resolution of the boundary layer; this choice was driven by the aim to find the best set-up to perform reliable LES simulations of internal combustion engines, where the correct mesh resolution near the walls implies a number of computational cells that is not affordable with the common computational resource limits.

Simulations have been carried out by either the dynamic Smagorinsky SGS model [6] and by the WALE model [12], which were implemented by the authors in the code. While the former does not require the tuning of any parameter, the constant in the WALE model has been set to $C_{\mathrm{w}}=0.58$ [12].

Advective outflow was imposed at the outlet boundary, no-slip conditions were set on the solid walls. Side surfaces were assumed as periodic. No wall-functions were used. Fields have been averaged both in time and space along the spanwise direction. Time averaging started after two flow-through times and lasted for three additional flow-through times.

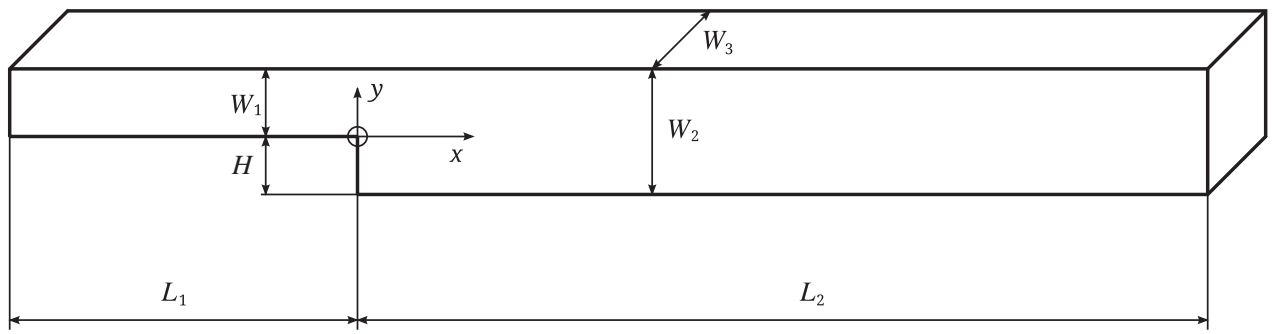

Figure 1. Schematic of the BFS geometry simulated [4]; $L_{1}=0.508 \mathrm{~m} ; L_{2}=1.524 \mathrm{~m} ; W_{1}=0.0762 \mathrm{~m} ; W_{2}=0.127 \mathrm{~m}$; $W_{3}=0.015 \mathrm{~m}$ and $H=0.0508 \mathrm{~m}$. 


\subsection{Results}

Comparisons between experimental and simulations at eight different measuring locations along the BFS are shown in Figure 2. In the upper row, results for the coarser mesh are reported. In the separation region $(x / H>0)$, the dynamic Smagorinsky model seems to have a slightly higher accuracy in predicting the mean flow, whereas the WALE model seems to behave a little worse. Such a trend is inverted in the finer mesh (bottom row), where the WALE model shows a better agreement with the experimental data. In particular, for the fine mesh the dynamic Smagorinsky model provides an asymmetric mean velocity profile in the inlet channel (negative values of $x$ ). Since the dynamic procedure for calculating the constant is numerically unstable, the implementation included an algorithm to clip the constant in order to avoid negative values of the modelled viscosity. On the other hand, clipping may lead to non-physical values of viscosity and the convergence of the solver may become less accurate. In this case, it has been verified that clipping was widely applied near the lower wall of the inlet channel, despite wall-resolution being the same adopted at the upper wall and the numerical set-up was the same for all the simulations. This statement is confirmed by the residuals of the solution of the conservation of mass that are higher when the dynamic Smagorinsky model is used. Despite the asymmetric profile disappearing by using a longer inlet channel or by a different set-up of the parameters of the solver, this was not done in the present work: the aim was to analyse the performance of the two SGS models working with the set-up adopted for the ICE simulation.

The near-wall profile at $x / H=-3$ is plotted against the theoretical law-of-the-wall [19] in Figure 3(a) and 3(b), for the coarse and fine mesh, respectively. The disagreement is not surprising since the mesh size is, in both cases, too coarse to correctly resolve near-wall turbulence dynamics. However, the WALE model better fits the logarithmic part of the curve, though the plot appears shifted upward by a significant amount. Instead, the dynamic Smagorinsky model lacks for the logarithmic part of the profile, especially in the case of Figure 3(b). The different near-wall velocity profiles between dynamic Smagorinsky and WALE can be explained by looking at Figure 4, which shows the SGS viscosity trend into the boundary layer. For both models, $v_{\text {sgs }}$ reaches very low
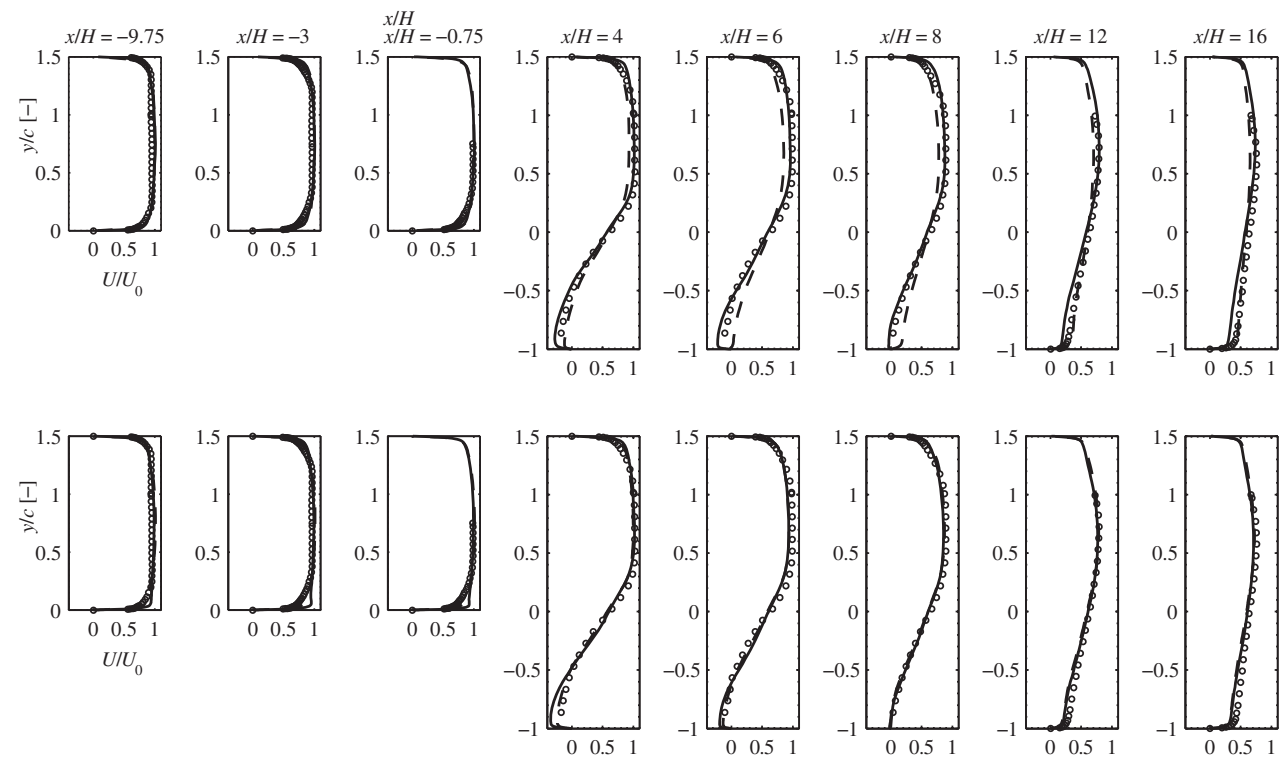

Figure 2. Comparison of mean velocity profiles inside the domain. First row: coarse mesh; second row: fine mesh. o, experimental data; -,-dynamic Smagorinsky and --, WALE. 
(a)

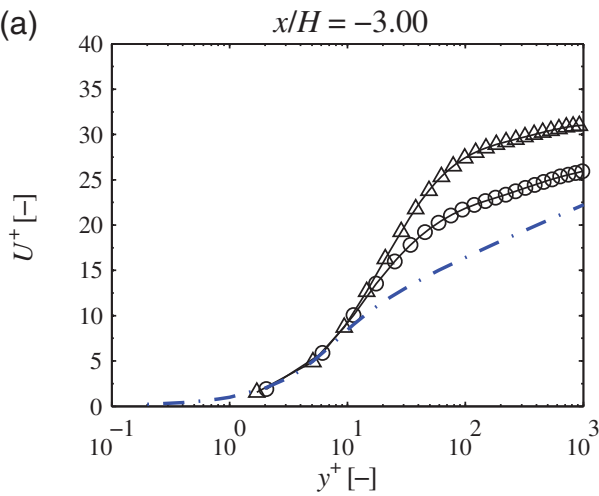

(b)

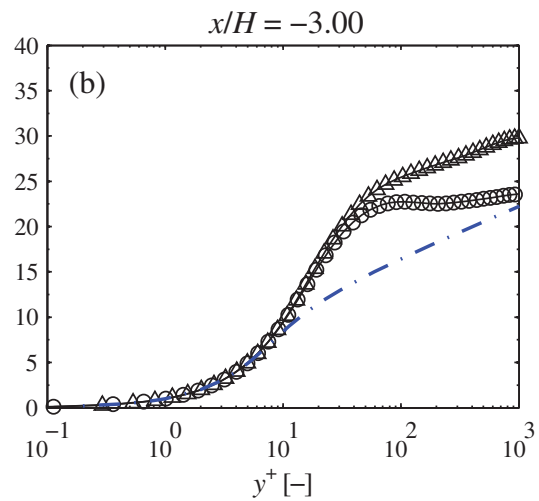

Figure 3. Near-wall velocity profiles for both cases in the channel region before separation $(x / H=-3)$. (a) Coarse mesh, (b) fine mesh. $-\circ-$, Dynamic Smagorinsky; $-\_$, WALE and $-\cdot-$, law of the wall.
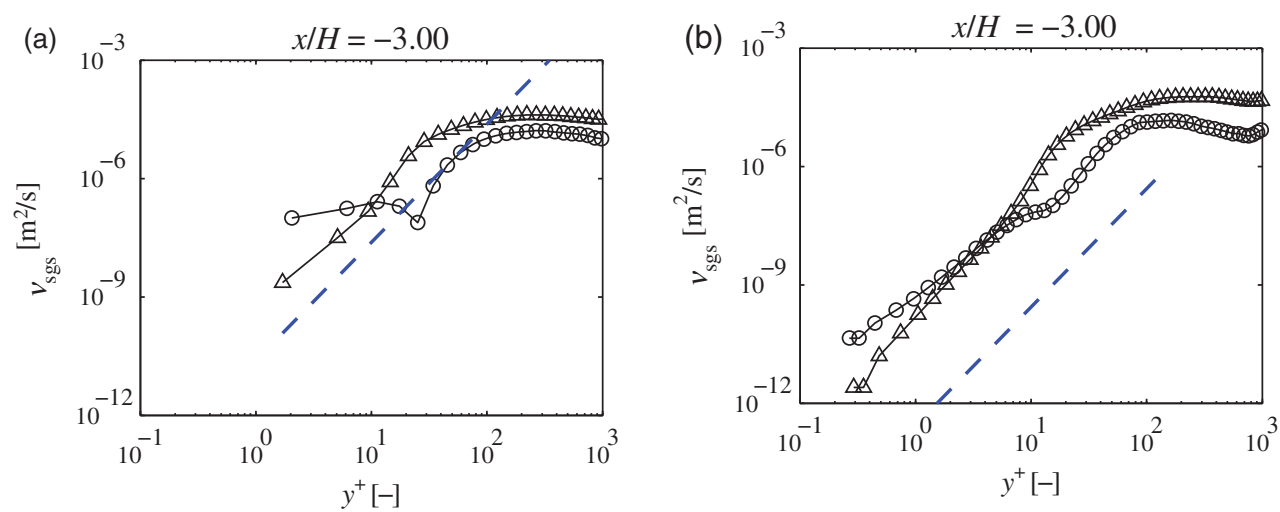

Figure 4. Near-wall SGS viscosity for both cases in the channel region before separation $(x / H=-3)$. (a) Coarse mesh, (b) fine mesh.-०-, Dynamic Smagorinsky; $-\backsim$, WALE and $---y^{3}$, slope.

values as approaching the wall, but the sole WALE model has the correct $y^{3}$ behaviour, whereas the viscosity obtained by the dynamic Smagorinsky decreases with a smoother slope.

Finally, root mean squared values (RMS) of streamwise fluctuations $\langle\bar{u} \bar{u}\rangle$ are plotted in Figure 5 against HWA measurements. When the coarser mesh is used (upper row), the WALE model does not give good predictions of $\langle\bar{u} \bar{u}\rangle$ in the separated flow region, whereas the dynamic Smagorinsky model correctly captures the measured fluctuations. With the fine mesh, both models produce comparable and good results (bottom row).

\section{Cold engine flow}

\subsection{Case set-up}

In order to investigate the performance of the WALE model when applied to an engine flow case, the configuration of Figure 6 has been simulated. The computational domain is an axis-symmetric sudden expansion from $d=34 \mathrm{~mm}$ to $D=120 \mathrm{~mm}$; a centred poppet valve is mounted through the expansion with a fixed lift of $10 \mathrm{~mm}$. The gas flowing through the valve forms an annular jet into the cylinder that produces a large toroidal recirculation region between the cylinder head and the walls. Reynolds number (referred to $d$ ) is 30,000 , corresponding to a bulk velocity of 

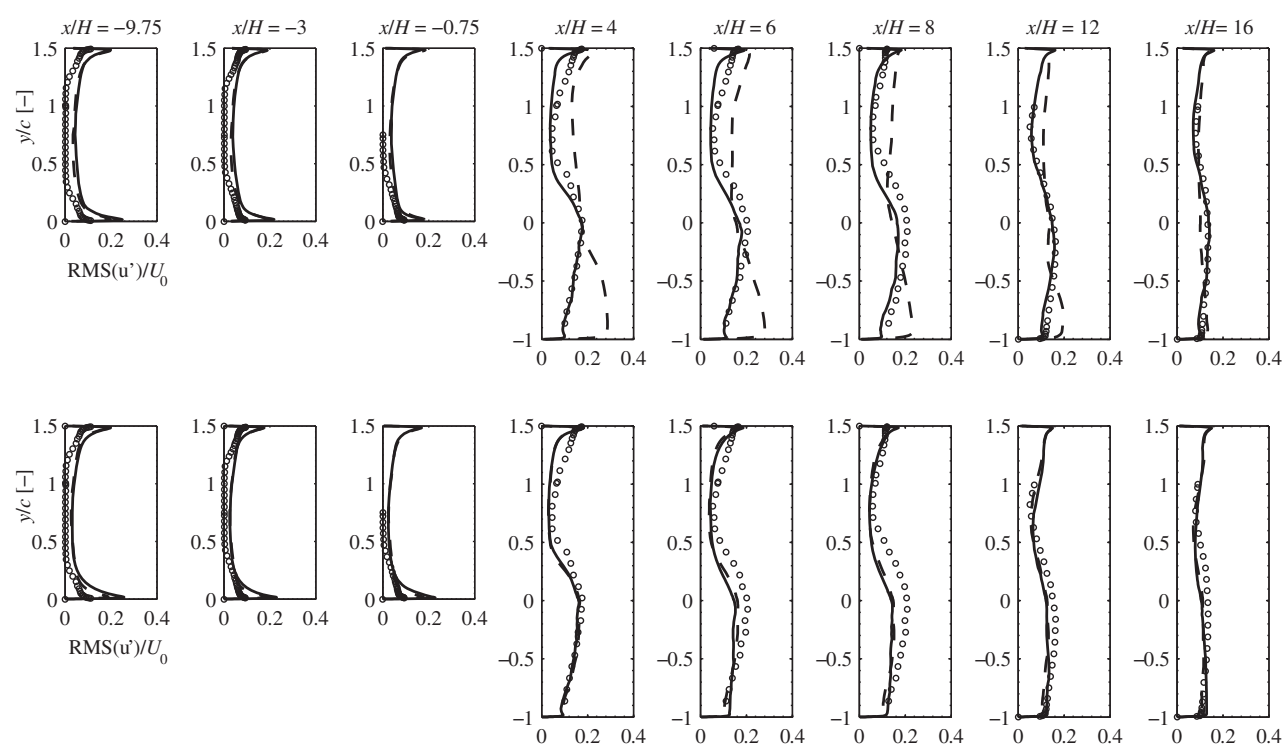

Figure 5. Comparison of RMS streamwise fluctuations inside the domain. First row: coarse mesh; second row: fine mesh. o, experimental data; - , dynamic Smagorinsky and - - , WALE.

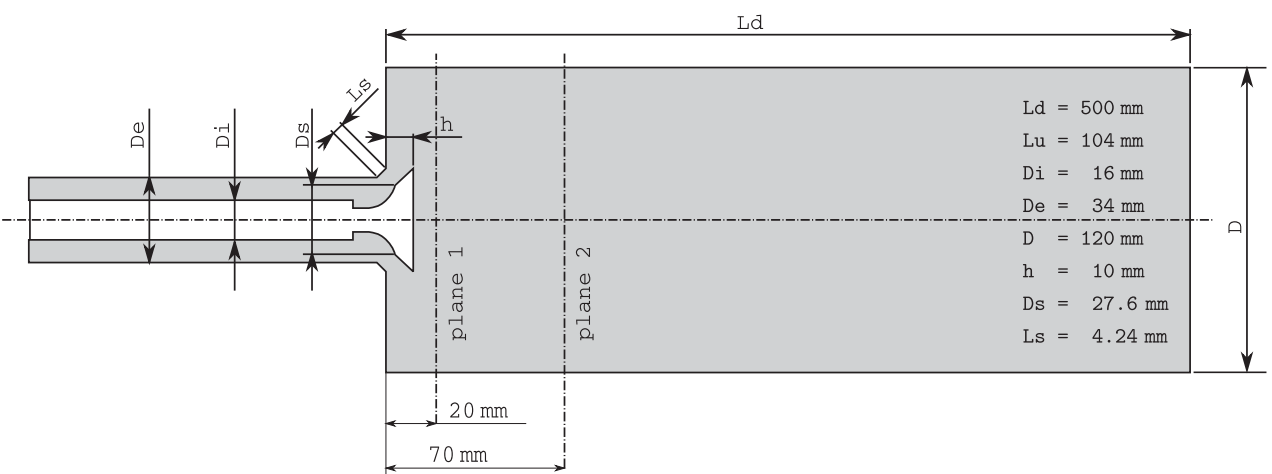

Figure 6. Two-dimensional schematization for the axial symmetric piston-cylinder assembly of [21], including the coordinate system.

$U_{0}=65 \mathrm{~m} / \mathrm{s}$; Mach number through the valve curtain area is lower than 0.2 , thus justifying the incompressibility hypothesis.

Two different meshes have been used in this stage. Both of them are fully hexahedral structured grids with wall refinement: the coarse mesh had about 1.4 million cells $\left(n^{+}=200, \Delta x_{r}^{+}=10\right.$, $\left.\Delta x_{t g}^{+}=500\right)$, whereas the finer mesh had about 13 million cells $\left(n^{+}=200, \Delta x_{r}^{+}=5, \Delta x_{t g}^{+}=\right.$ 500). $n^{+}, \Delta x_{t g}^{+}, \Delta x_{r}^{+}$are the resolutions along the cylinder axis, the tangential and the radial direction, respectively. A detail of the 14 million cell grid is shown in Figure 7.

Since the largest part of turbulence is produced by the shear layer downstream of the valve, generating a turbulent velocity field at the inflow is of minor importance in this case. Thus, uniform boundary condition on the velocity $U=U_{0}$ has been imposed at the inlet. Like the BFS case, all walls have been modelled as no-slip surfaces and advective outflow has been used as the outlet boundary condition. 


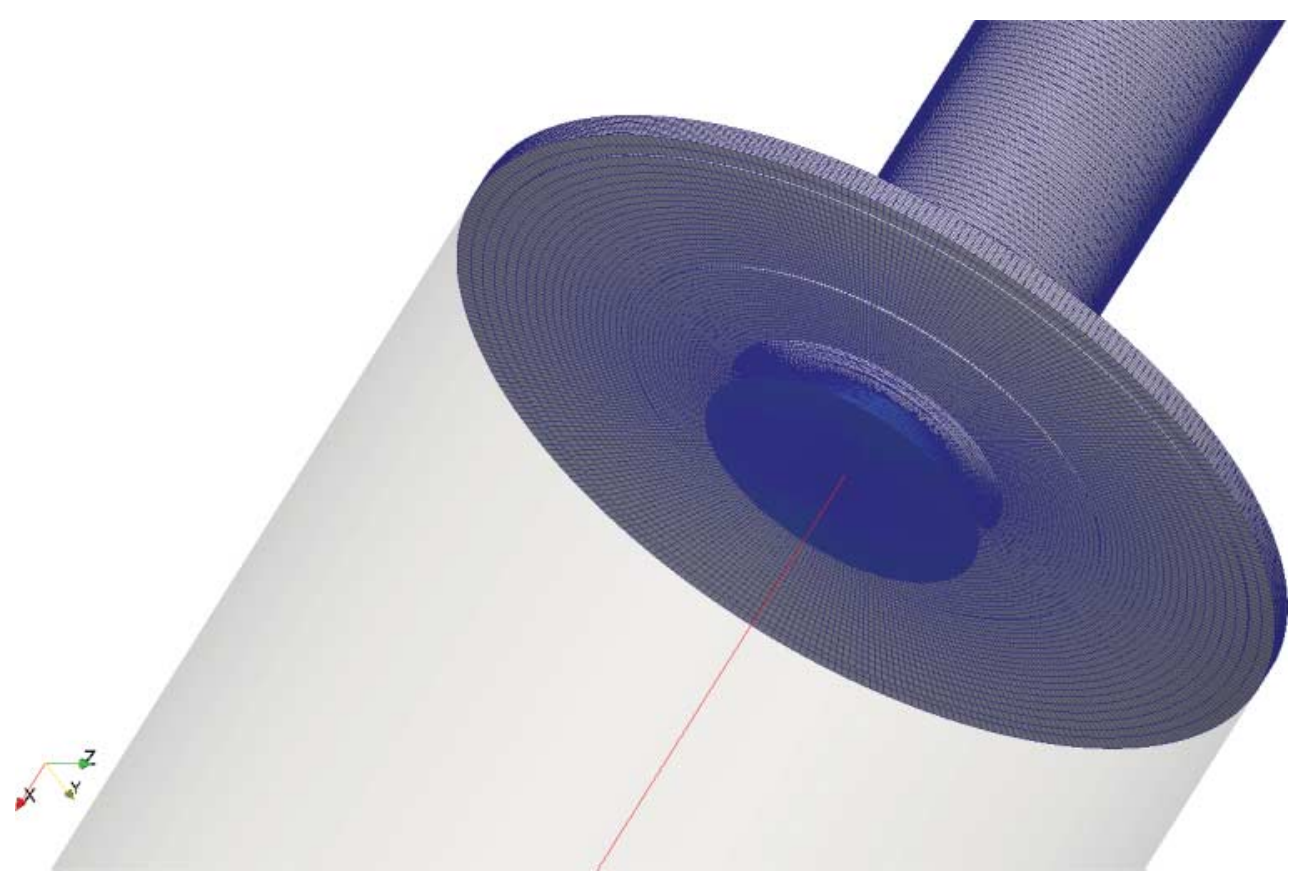

Figure 7. Detail of the computational 14 million cell grid ('fine grid') used for cold cylinder flow simulations.

\subsection{Results}

Results for mean velocity and RMS fluctuations are shown in Figure 8 for both meshes. Samples were taken on two planes represented in Figure 6, respectively, at 20 and $70 \mathrm{~mm}$ from the cylinder head.

In Figure 8(a) and 8(d), the mean velocity profiles are plotted. For each radial position, instantaneous velocity has been averaged both in time and in the circumferential direction, to reduce the number of flow-through times to simulate. The agreement between numerical results and LDA measurements is good on both the $20 \mathrm{~mm}$ plane (Figure $8(\mathrm{a})$ ) and the $70 \mathrm{~mm}$ plane (Figure 8(d)), even though the coarser mesh exhibits a better accuracy. This counterintuitive fact will be analysed later.

A similar trend can be seen with respect to the axial fluctuations in Figure 8(b) and 8(e), where the curves computed with the finer mesh depart from the expected values by a significant amount, even though the overall pattern is much the same.

On the other hand, there is a strong overprediction of circumferential fluctuations on the $20 \mathrm{~mm}$ plane by both the coarse and the fine grid (Figure 8(c)), while on the $70 \mathrm{~mm}$ plane such a discrepancy appears to be reduced, probably because of the lower magnitude of fluctuations themselves.

The better accuracy of the solution obtained by the coarse mesh, in comparison with the fine one, is probably due to a reciprocal cancellation of modelling errors and numerical errors. In fact, for the WALE constant $C_{\mathrm{w}}$, an empirical value has been adopted, which was tuned by Nicoud and Ducros [12] for homogeneous isotropic turbulence, and validated only for channel flow. There is a possibility that, for this kind of flow, a different estimate of $C_{\mathrm{w}}$ has to be used. On the other hand, numerical viscosity might have been introduced into the flow by the effects of flux limiters [8] and mesh non-orthogonality [10]. This is consistent with the observation that the finer mesh (which has lower numerical dissipation) produces higher fluctuations, and this also suggests that a lower value of $C_{\mathrm{w}}$ might be needed. 

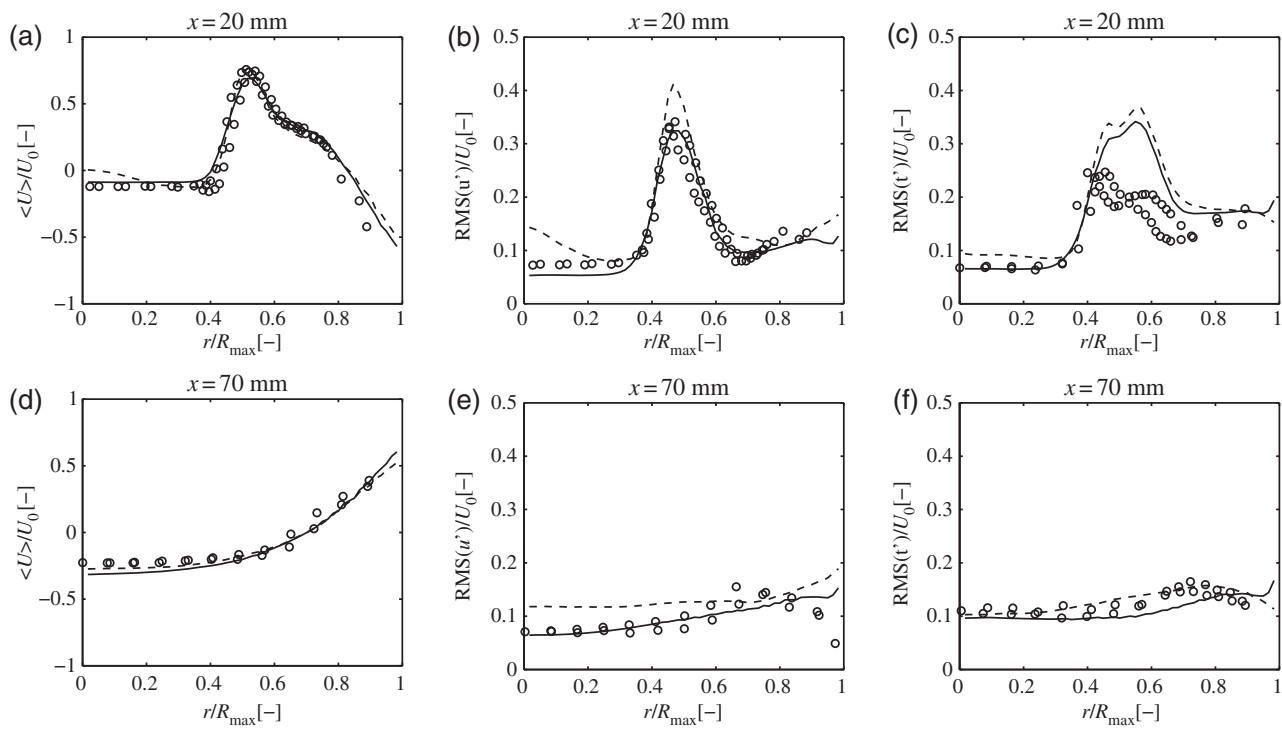

Figure 8. Comparison of the velocity profiles between the LES simulation and the LDA measurements. Plane $x=20 \mathrm{~mm}$ : (a) mean axial velocity, (b) mean axial velocity fluctuations and (c) mean tangential velocity fluctuations. Plane $x=70 \mathrm{~mm}$ : (d) mean axial velocity, (e) mean axial velocity fluctuations and (f) mean tangential velocity fluctuations. - , coarse mesh; - -, fine mesh and o, experiments. Two-dimensional schematization for the axial symmetric piston-cylinder assembly of [21], including coordinate system, is shown in the top.

\section{Conclusions}

In this work, the development of the dynamic Smagorinsky and of the WALE model for incompressible flows is presented and it is applied to steady simulations of ICE. Model validation has been performed on a BFS, which is a good validation test case for engines, since it includes phenomena that are similar to the ones occurring during the intake stroke of the engine, when air flows to the combustion chamber through the valve ports. Later, the WALE model was applied to a simplified engine geometry. Even though the overall agreement with experiments looks satisfying, simulations carried out with a coarser mesh showed better accuracy. Since this phenomenon can be ascribed to a modelling error, namely too low a value of the constant $C_{\mathrm{w}}$, the use of a dynamic version of the WALE SGS [1] model may represent a general solution to this problem.

Since experimental data on the engine-like geometry were available at flow Mach numbers lower than 0.3, the incompressible formulation of the models was used. The models and the set-up described in this paper are intended to be for cold flow steady simulations of ICE by the open-source CFD code OpenFOAM ${ }^{\circledR}$ and the work represents a step towards the LES simulation of a real engine cycle. In this context, the current work is focussed on the implementation of a parallel algorithm for the moving mesh with topological changes for ICE simulation and of SGS models for compressible flows.

\section{Acknowledgements}

Computational resources for this project have been made available by CINECA (Bologna, Italy) through the ISCRA (Italian Super-Computing Resource Allocation) framework, project LES4ICE.

\section{References}

[1] H. Baya Toda, K. Truffin, and F. Nicoud, Is the dynamic procedure appropriate for all SGS models? V European Conference on Computational Fluid Dynamics, ECCOMAS CFD, 2010. 
[2] D. Chapman, Computational aerodynamics development and outlook, AIAA J. 17 (1979), pp. 1293-1313.

[3] L. Davidson, Using isotropic synthetic fluctuations as inlet boundary conditions for unsteady simulations, Adv. Appl. Fluid Mech. 1 (2007), pp. 1-35.

[4] J. Eaton, J. Johnston, and R. Westphal, Experimental study offlow reattachment in a single-sided sudden expansion, Contractor report 3765, NASA Ames Research Center, 1986.

[5] J.H. Ferziger and M. Perić, Computational Methods for Fluid Dynamics, Springer, Berlin, 1997.

[6] M. Germano, U. Piomelli, P. Moin, and W.H. Cabot, A dynamic subgrid-scale eddy viscosity model, Phys. Fluids A 3 (1991), pp. 1760-1765.

[7] C.J. Greenshields, H.G. Weller, L. Gasparini, and J.M. Reese, Implementation of semi-discrete, non-staggered central schemes in a colocated, polyhedral, finite volume framework, for high-speed viscous flows, Int. J. Numer. Methods Fluids 63 (2010), pp. 1-21. Available at http://dx.doi.org/10.1002/fld.2069.

[8] C. Hirsch, Numerical Computation of Internal and External Flows, 2nd ed., Butterworth-Heineman, Oxford, 2007.

[9] H. Jasak, Error analysis and estimation in the finite volume method with applications to fluid flows, Ph.D. thesis, Imperial College, University of London, 1996.

[10] F. Juretić and A.D. Gosman, Error analysis of the finite-volume method with respect to mesh type, Numer. Heat Transf. B, Fundam. 57 (2010), pp. 414-439.

[11] A. Montorfano, F. Piscaglia, and G. Ferrari, Inlet boundary conditions for incompressible LES: A comparative study, Math. Comp. Modell. 57 (2013), pp. 1640-1647. doi:10.1016/j.mcm.2011.10.077.

[12] F. Nicoud and F. Ducros, Subgrid-scale stress modelling based on the square of the velocity gradient tensor, Flow Turbul. Combust. 62 (1999), pp. 183-200.

[13] OpenCFD, OpenFOAM Programmer's Guide, OpenCFD Ltd., London, 2004.

[14] OpenCFD, OpenFOAM User's Guide, v2.1.1, OpenCFD Ltd., London, 2012.

[15] S.B. Pope, Turbulent Flows, Cambridge University Press, Cambridge, 2001.

[16] P. Sagaut, Large Eddy Simulation for Incompressible Flows: An Introduction, Scientific Computation, SpringerVerlag, Berlin, 2006

[17] H. Schlichting and K. Gersten, Boundary Layer Theory, 8th ed., Springer, Berlin, 2000.

[18] J. Smagorinsky, General circulation experiments with the primitive equations. I: The basic experiments, Mon. Weather Rev. 91 (1963), pp. 99-165.

[19] D.B. Spalding, A single formula for the law of the wall, Trans. ASME J. Appl. Mech. E 28 (1961), pp. $455-458$.

[20] G.R. Tabor and M.H. Baba-Ahmadi, Inlet conditions for large eddy simulation: A review, Comput. Fluids 39 (2010), pp. 553-567.

[21] L. Thobois, G. Rymer, T. Soulères, and T. Poinsot, Large-eddy simulation in IC engine geometries, SAE Paper n. 2004-01-1854, SAE world Congress \& Exhibition, 2004.

[22] E.R. Van Driest, On turbulent flow near a wall, J. Aeronaut. Sci. 23 (1956), pp. 1007-1011, 1036.

[23] H.K. Versteeg and W. Malalasekera, An Introduction to Computational Fluid Dynamics, Longman Scientific and Technical, Harlow, UK, 1995. 